\title{
Problem of false indicators for kimberlites and lamproites (on the example of chromites).
}

Afanasiev, V.P., Pokhilenko, N.P., Logvinova, A.M., Yefimova, E.S.

Institute of Mineralogy and Petrography, Siberian Branch of Russian Academy of Sciences, Novosibirsk, 630090, Russia

In 1972, a great deal of Cr-spinels were found near the town of Mirny, Western Yakutia. Compositionally, they were similar to kimberlitic varietes but drastically differed from them in morphology. Up to $30 \%$ of these spinellide corresponded in composition to chromite inclusions in diamonds. This suggested the existence of unknown bodies with an unusual morphological variety of $\mathrm{Cr}$-spinels, and some geologists believe that these bodies are diamondiferous kimberlites. Later on, similar $\mathrm{Cr}$-spinels were found in placers throughout the Yakutian diamondiferous province, in the Arkhangelsk Region, in many places beyond Russia, and on other continents. Nevertheless, none of the kimberlite sources of these $\mathrm{Cr}$-spinels has been found yet. In all the known kimberlites and lamproites $\mathrm{Cr}$-spinels are of different morphology. The challenge was to establish the type of sources of the new variety of $\mathrm{Cr}$-spinels. The crystalline inclusions present in these $\mathrm{Cr}$-spinels are olivine, Opx, Cpx, mica, amphibole, and plagioclase. Investigations in this direction permitted us to demonstrate that in morphology they are completely analogous to and in composition overlaps the Cr-spinels from ultrabasic intrusions of the type of Kempirsai, Chad, Konder, and many other plutons (see Table). This suggests that the new variety of $\mathrm{Cr}$-spinels originated from intrusions of ultrabasic rocks. These intrusions are not diamondiferous, but close compositions of their $\mathrm{Cr}$-spinels with those from kimberlites and lamproites (with morphology ignored) are misleading in exploration for diamonds. Geologists used these Cr-spinels as indicators of kimberlites or lamproites and, to follow them, pursue diamonds with no result. Therefore, in exploration for kimberlites and lamproites the ultrabasic $\mathrm{Cr}$-spinels may play the role of false indicators.

Each ultrabasic intrusion contains $\mathrm{Cr}$-spinels of specific composition, but these compositions overlap with kimberlitic (lamproitic) varieties. Therefore, the main difference between two types of $\mathrm{Cr}$ spinels mantle-derived type from kimberlites (lamproites) and crust-derived type from ultrabasic intrusions lies in morphology. Differences in morphology are, probably, due to differences in processes of crystallization: under conditions of the upper mantle for the first type and under conditions of the crust, for the second. The $\mathrm{Cr}$-spinels from intrusions of ultrabasic rocks are significantly more widespread in sedimentary collectors than the Cr-spinels from kimberlites and lamproites. Therefore, the problem of false indicators has come into importance in searching for diamonds. On the basis of chemical composition only, one cannot always discriminate between the "mantle" and the "crustal" Cr-spinels, but the additional study of morphology permits them to be discriminated in a reliable way.

\section{References}

Sobolev, N.V., Pokhilenko, N.P., Lavrentiev, Yu.G., Usova, L.V., 1975, Compositional peculiarities of chromespinelides from diamonds and kimberlites of Yakutia (in Russian): Geologiya i Geofizika, v.16(11), p. 7-24. English Translation: Soviet Geology and Geophisics, v.16(11), p.7-24.

Simonov V.A., 1993, Petrogenesis of ophiolites: Novosibirsk, Trudy UIGGM, v. 816, p. 248. 


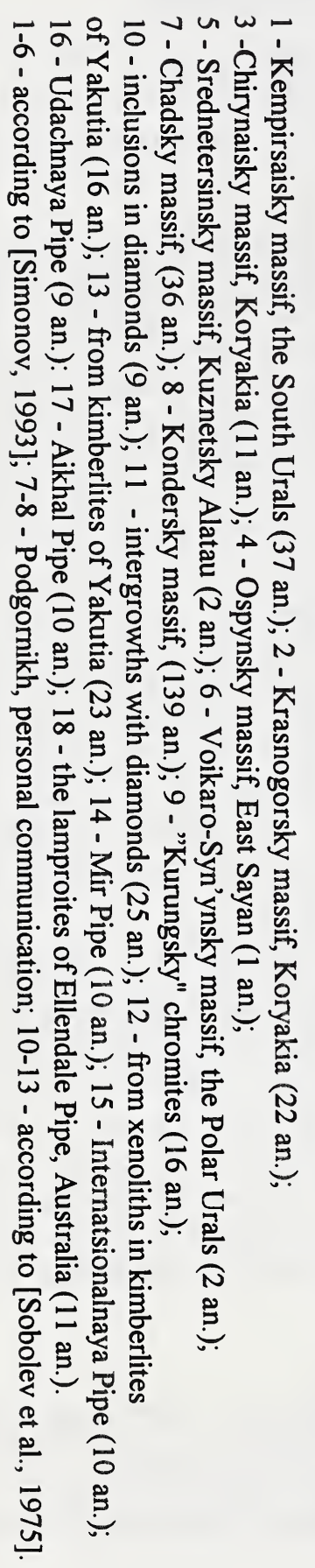

\begin{tabular}{|c|c|c|c|c|c|c|c|c|c|c|c|c|c|c|c|}
\hline$F$ & ज小 & $\bar{n}$ & $\vec{\Phi}$ & $\vec{\omega}$ & $\bar{N} \equiv$ & $=0$ & 0 & $\infty$ & 4 & a) & u. & $\Delta \mid c$ & | & - & ไె. \\
\hline : & 히항 & $=$ & 이 & $\vec{i}$ & $\vec{\infty}$ & ०) & 10 & 인 & 임 & ㅇ. & 인. & $=$ & & $=0$ & $\overrightarrow{0} \times 1$ \\
\hline & 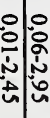 & $\hat{n}$ & $\begin{array}{l}0 \\
\stackrel{0}{0} \\
+ \\
N \\
\triangleq\end{array}$ & $\begin{array}{c}0 \\
\vdots \\
\vdots \\
\vdots \\
\vdots\end{array}$ & 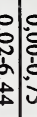 & 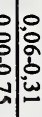 & $\mid \begin{array}{l}0 \\
0 \\
0 \\
0 \\
0 \\
0 \\
0\end{array}$ & $\mid \begin{array}{l}0 \\
\dot{\omega} \\
\dot{\omega} \\
\sigma\end{array}$ & $\begin{array}{l}0 \\
0 \\
\vdots \\
\vdots \\
\\
0\end{array}$ & $\begin{array}{l}0 \\
\vec{y} \\
0 \\
w \\
0\end{array}$ & $\begin{array}{l}0 \\
0 \\
0 \\
0 \\
0 \\
0 \\
\infty\end{array}$ & & 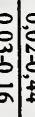 & 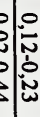 & $\underset{\mathrm{g}}{\mathrm{g}}$ \\
\hline$|\vec{\omega}|$ & 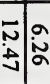 & 可 & $\mid$ & $\bar{\omega}:=$ & $\forall$ & $\stackrel{\Delta}{\circ}$ & $\mid \begin{array}{l}\infty \\
\infty \\
\infty\end{array}$ & $\mid$ & के & iे & : & $\vec{\omega}$ & $\begin{array}{l}\infty \\
\omega\end{array}$ & 0 & ${ }_{0}^{2} \times 1$ \\
\hline $\mid \begin{array}{l}u \\
\tilde{u} \\
\hat{n} \\
\hat{0} \\
\dot{0} \\
\tilde{o}\end{array}$ & 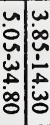 & 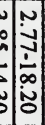 & $\begin{array}{l}u \\
\dot{0} \\
\dot{\omega} \\
\stackrel{\alpha}{\delta}\end{array}$ & $\begin{array}{l}\mathbf{N} \\
\vec{u} \\
\hat{a} \\
\vdots \\
0 \\
0\end{array}$ & 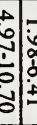 & 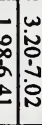 & 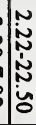 & $\begin{array}{l}0 \\
\dot{0} \\
\dot{b} \\
\dot{\vec{b}}\end{array}$ & \begin{tabular}{|l|}
$\omega$ \\
$\tilde{N}$ \\
$\tilde{N}$ \\
$\dot{\infty}$ \\
$\tilde{\omega}$ \\
$\infty$
\end{tabular} & \begin{tabular}{c|}
$\infty$ \\
0 \\
$\dot{\omega}$ \\
$\vdots$ \\
$\vdots$ \\
0 \\
0
\end{tabular} & 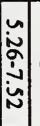 & & 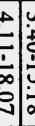 & 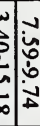 & 营 \\
\hline $\begin{array}{ll}0 \\
\infty \\
\infty \\
\infty\end{array}$ & $\begin{array}{l}u \\
\dot{0} \\
\dot{0}\end{array}$ & $\vec{\Delta}$ & $\begin{array}{l}n \\
\infty \\
\vdots \\
\sim\end{array}$ & $\begin{array}{ll}\omega \\
\omega\end{array}$ & & & & 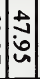 & $\underset{\infty}{\simeq}$ & 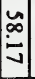 & $\frac{a}{\sim}$ & & $\begin{array}{ll}\infty \\
0 \\
0\end{array}$ & $\begin{array}{l}\hat{0} \\
0\end{array}$ & 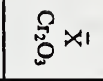 \\
\hline $\begin{array}{l}n \\
\vdots \\
0 \\
0 \\
\hat{0} \\
0 \\
0\end{array}$ & 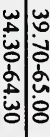 & 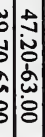 & 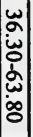 & 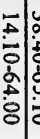 & $\begin{array}{l}\omega \\
\infty \\
\vdots \\
\vdots \\
\vdots \\
\vdots \\
0\end{array}$ & 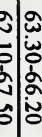 & $\hat{0}$ & 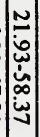 & $\begin{array}{l}\vec{\omega} \\
\dot{\omega} \\
\hat{\omega} \\
\ddot{\alpha} \\
\ddot{\alpha} \\
\alpha\end{array}$ & $\begin{array}{l}\tilde{\omega} \\
\dot{u} \\
\hat{n} \\
\hat{n} \\
\vec{v}\end{array}$ & 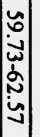 & & 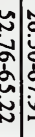 & 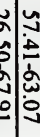 & 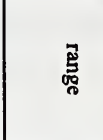 \\
\hline 함 & $\stackrel{\vec{\omega}}{\omega} \vec{\omega}$ & aे & $\dddot{\infty}$ & $\begin{array}{l}-0 \\
\text { in } \\
\text { : }\end{array}$ & aे? & w. & \& & 啇 & जे & $\underset{\omega}{\omega}$ & $\vec{\sim}$ & : & iे) & w & $0_{0}^{3} \times 1$ \\
\hline$\left|\begin{array}{c}\dot{0} \\
\vdots \\
\dot{\infty} \\
\dot{\omega} \\
0\end{array}\right|$ & 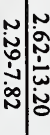 & 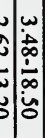 & 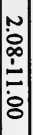 & 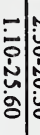 & : & 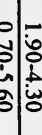 & 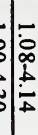 & 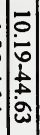 & 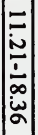 & $\begin{array}{l}\overrightarrow{\hat{n}} \\
\hat{\tilde{N}} \\
\dot{0} \\
\dot{\mathrm{N}} \\
\hat{\mathrm{N}}\end{array}$ & $\mid \begin{array}{c}\omega \\
\dot{\omega} \\
\dot{t} \\
\hat{\sigma}\end{array}$ & & . & 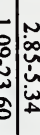 & 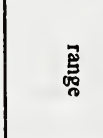 \\
\hline ज्ञ: & जे| & t. & $\vec{\omega}$ & 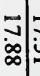 & जut & $\vec{\alpha}$ & : & $\frac{5}{\square}$ & जu & $\stackrel{\vec{N}}{\sim}$ & $\mid \begin{array}{l}\infty \\
\infty \\
\infty\end{array}$ & : & & है। & $8 \times 1$ \\
\hline $\mid \begin{array}{l}0 \\
0 \\
0 \\
0 \\
\\
0\end{array}$ & 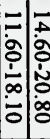 & 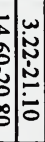 & \begin{tabular}{l}
$\overrightarrow{0}$ \\
$\dot{0}$ \\
0 \\
$\vdots$ \\
\hdashline \\
0 \\
0
\end{tabular} & 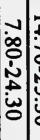 & : & \begin{tabular}{l|l}
0 \\
$\vdots$ \\
0
\end{tabular} & 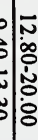 & 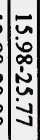 & 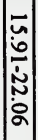 & 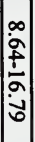 & 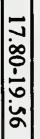 & & 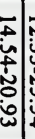 & 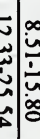 & $\underset{\text { 喿 }}{\vec{\sigma}}$ \\
\hline $\mid \begin{array}{c}\mid \\
\\
\dot{\infty} \\
\end{array}$ & $\bar{N}$ & $=$ & $\bar{\omega}$ & $\underset{N}{\omega}$ & సे & $=$ & $\overrightarrow{0}$ & w & $\left|\begin{array}{l}\infty \\
\infty \\
\omega\end{array}\right|$ & $\left|\begin{array}{l}\omega \\
\dot{a}\end{array}\right|$ & $\stackrel{0}{N}$ & $\tilde{\sim}$ & 하: & $8 \mid$ & $\frac{3}{8} \times 1$ \\
\hline$=$ & $\overline{0}$ & 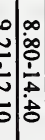 & 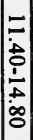 & $\begin{array}{l}0 \\
0 \\
\dot{\omega} \\
\dot{0} \\
\dot{0}\end{array}$ & îl & 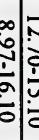 & 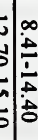 & 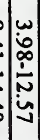 & $\begin{array}{l}.1 \\
\dot{0} \\
\dot{0}\end{array}$ & 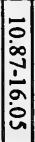 & $\begin{array}{l}\infty \\
\dot{w} \\
\infty \\
\phi \\
\dot{0} \\
\infty \\
\alpha\end{array}$ & & : & 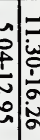 & 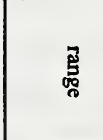 \\
\hline 0 & $\infty$ & : & & : & & i̊ & ת & 离 & : & & $\mid \begin{array}{l}0 \\
\vdots \\
\end{array}$ & : & & 0 & $3 \times 1$ \\
\hline 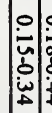 & 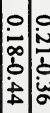 & bे & & $\mid$\begin{tabular}{l}
0 \\
0 \\
0 \\
0 \\
\hdashline \\
-1
\end{tabular} & & 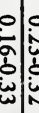 & & 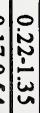 & $\begin{array}{l}0 \\
\dot{\sim} \\
\vdots \\
\dot{\infty} \\
\dot{\infty} \\
N\end{array}$ & $\begin{array}{l}0 \\
\dot{\omega} \\
\infty \\
b \\
\dot{\omega} \\
0\end{array}$ & 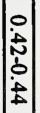 & & 家 & & 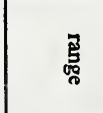 \\
\hline
\end{tabular}

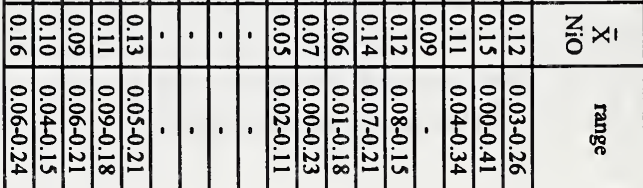

อั 